\title{
Slower Can Be Faster: The iRetis Incremental Model Tree Learner
}

\author{
Denny Verbeeck ${ }^{1}$ and Hendrik Blockeel ${ }^{1,2}$ \\ 1 Department of Computer Science, KU Leuven \\ 2 Leiden Institute of Advanced Computer Science, Leiden University \\ $\{$ denny.verbeeck, hendrik.blockeel\}cs.kuleuven.be
}

\begin{abstract}
Incremental learning is useful for processing streaming data, where data elements are produced at a high rate and cannot be stored. An incremental learner typically updates its model with each new instance that arrives. To avoid skipped instances, the model update must finish before the next element arrives, so it should be fast. However, there can be a trade-off between the efficiency of the update and how many updates are needed to get a good model. We investigate this trade-off in the context of model trees. We compare FIMT, a state-of-the-art incremental model tree learner developed for streaming data, with two alternative methods that use a more expensive update method. We find that for data with relatively low (but still realistic) dimensionality, the most expensive method often yields the best learning curve: the system converges faster to a smaller and more accurate model tree.
\end{abstract}

\section{Introduction}

Data stream mining is a subfield of data mining that is concerned with analyzing streaming data. There are multiple types of learning tasks in this setting. We here focus on the task of learning a predictive model for the data elements in the stream; that is, given a stream of elements of the form $\left(\mathbf{x}_{i}, y_{i}\right) \in \mathcal{X} \times \mathcal{Y}$, learn a model $M: \mathcal{X} \rightarrow \mathcal{Y}$ that predicts $y$ from $\mathbf{x}$. This task is identical to standard predictive learning, except for one additional constraint: each data element can be looked at only once (and briefly). This setting is ubiquitous in the context of "big data"; for instance, sensors produce large amounts of data at a high pace, and often these data must be analyzed online.

There has been a significant amount of work on adapting several types of data mining methods to the stream context. Commonly, methods that construct predictive models are adapted such that they become incremental: that is, they start with an initial model and update their model each time a new data element is observed. Ideally, the model learned in this way converges to the model that would be learned if all data were stored in a database and, next, a regular learner were used. The incremental learner has the advantage that some model is available at any point in time, well before all data have arrived.

Ideally, the update step is computationally light enough that it can be performed in the time interval between the arrival of two data elements. If this 
is not the case, then the learner has to skip elements that arrive before it is ready to handle another input, which may slow down learning. However, more efficient update methods may result in lower-quality updates, which may lead to more updates being needed to achieve the same model quality. Moreover, a more expensive update does not necessarily result in skipped instances: as long as it finishes before the next example arrives, the overall learning time is not affected, only the idle time between instances is reduced.

Based on these considerations, we investigate incremental model tree learners. Ikonomovska et al. [5] have proposed an incremental model tree learner, FIMT, that can be considered the state of the art in model tree learning from streaming data. In this paper, we propose two alternative model tree learners for streaming data, iRetis and iMauve. Both methods use the same approach for keeping statistics and deciding the best time to split the tree compared to FIMT. However they employ a more complicated split heuristic and model update mechanism, which should enable these methods to choose splits more intelligently. We experimentally evaluate how they compare to FIMT. We find that, for relatively low-dimensional data, the most complicated update method often gives the best learning curve: it converges faster to simpler and more accurate model trees.

Section 2 discusses related work and describes FIMT in detail. Section 3 describes the modifications that yield iRetis and iMauve. Section 4 reports on experiments, and Section 5 concludes.

\section{Background and Related work}

A decision tree is a rooted tree where each internal node contains a test and has one child for each outcome of the test. In the machine learning context, such a tree can be used as a predictive function: instances are sorted down the tree based on their test outcomes, and the leaf they end up in contains the prediction. Trees that make numerical predictions are called regression trees. While regression trees often contain a constant prediction in each leaf, variants exist that have a linear model in the leaf; these are called model trees [7]. A model tree defines a piecewise linear function.

Most decision trees learners learn the tree top-down $[2,8]$. They determine which test should be at the root of the tree by evaluating all possible tests on the whole dataset, choosing the best one (according to some heuristic), and splitting the data according to this test. They then repeat the procedure for the child nodes, and so on until leaves are obtained.

The above assumes access to the whole data set at any time. Incremental learners do not require this: they start with an empty model, and update this model each time they see a new example. By definition, they need to decide on which test to put in a node before seeing the whole dataset. But a test that seems optimal at this time may later turn out not to be. There are three ways of dealing with this: (1) choose a test that may yet turn out to be non-optimal, and repair the tree afterwards if necessary (by restructuring it or simply discarding part of the tree and learning it anew); (2) wait until there is enough statistical 
evidence to make it highly unlikely that the chosen test will turn out non-optimal later on; or (3) simply accept that a non-optimal tree is learned.

In their seminal work on VFDT ("very fast decision tree learner"), Domingos and Hulten [3] advocate the second approach. Using so-called Hoeffding bounds, they characterize under which conditions there is sufficient statistical evidence that the test that seems optimal at this point is equal to the one that would seem optimal when looking at the whole dataset. Since VFDT, multiple tree learners have adopted the use of Hoeffding bounds; these are called Hoeffding tree learners. It is in this seam of work that Ikonomovska et al. [5] have introduced Fast Incremental Model Trees (FIMT), an algorithm that incrementally learns model trees from streams, using Hoeffding bounds. As our work builds on it, we describe FIMT in detail in the following section.

\section{$2.1 \quad$ FIMT}

Like VFDT, FIMT starts off with an empty leaf, reads the examples from the stream as they arrive, and gradually splits leaves (turning them into an internal node) when there is sufficient evidence that the proposed split is optimal. To this aim, it keeps some statistics in each leaf, and for each incoming example the corresponding leaf is determined and its statistics updated. Sufficient statistics must be kept, taking into account that not only the tree structure, but also the linear model in the leaves must be learned incrementally. As the statistics are not expected to differ significantly between consecutive examples, FIMT considers a new split only once every $N_{\min }$ examples.

Splitting FIMT assumes numerical input attributes and uses tests of the form $X_{i}<c$ with $X_{i}$ an input attribute and $c$ a threshold; $c$ is also called a "split point". FIMT first finds the best split point for each attribute, and then ranks these attributes according to the Standard Deviation Reduction (SDR) measure, which for a split of $S$ into $S_{L}$ and $S_{R}$ is defined as:

$$
\begin{gathered}
S D R\left(S, S_{L}, S_{R}\right)=s d(S)-\frac{N_{L}}{N} s d\left(S_{L}\right)-\frac{N_{R}}{N} s d\left(S_{R}\right), \\
s d(S)=\sqrt{\frac{1}{N}\left(\sum_{i=1}^{N}\left(y_{i}-\bar{y}\right)^{2}\right)}=\sqrt{\frac{1}{N}\left(\sum_{i=1}^{N} y_{i}^{2}-\frac{1}{N}\left(\sum_{i=1}^{N} y_{i}\right)^{2}\right)}
\end{gathered}
$$

Equation (2) shows the SDR can be computed efficiently and incrementally, by maintaining the sum of (squared) $y$ values and the number of data points.

FIMT considers the ratio of the SDR values for the two best candidate splits, $r=S D R_{1} / S D R_{2}$, with $S D R_{1}$ the best and $S D R_{2}$ the second best SDR value. FIMT uses the Hoeffding probability bound [4] to state with confidence $1-\delta$ that the sample average $\bar{r}$ over the $N$ values for $r$, which have range $R$, is within distance $\epsilon$ of the true average:

$$
\epsilon=\sqrt{\frac{R^{2} \ln (1 / \delta)}{2 N}} .
$$


With confidence $1-\delta$, the upper bound of the true average of $r$ is $\bar{r}+\epsilon$. If this upper bound is below 1 , the true average of $r$ is below 1 (and $c_{a}$ is the best split) with confidence $1-\delta$, and the split is applied.

FIMT builds an Extended Binary Search Tree (E-BST) for the values of each attribute to efficiently maintain the necessary statistics for split quality calculations. The tree structure of an E-BST is like a regular BST, but each node maintains two arrays of statistics; one for attribute values less than or equal to the node value, and one for attribute values greater than the node value. A BST structure allows for $O(\log (n))$ average insertion time, and $O(n)$ worst case insertion time. When a leaf node of the regression tree is split, its E-BST structure is discarded, and two new structures are instantiated for the children of that node.

Leaf models FIMT maintains a linear perceptron without activation function in each leaf of the model tree. If each data point has $m$ attributes, the linear model will hold a weight vector $\mathbf{w}$ of length $m+1$, and the prediction $\hat{y}$ for a data point $\mathbf{x}$ is calculated as follows:

$$
\hat{y}=w_{0}+\sum_{i=1}^{m} x_{i} w_{i}
$$

The weights are updated with each arriving example using the Delta (a.k.a. Widrow-Hoff) rule:

$$
w_{i} \leftarrow w_{i}+\eta(\hat{y}-y) x_{i}^{\prime}, \quad i=1, \ldots, m
$$

with $x_{i}^{\prime}=\left(x_{i}-\bar{x}_{i}\right) /\left(3 \sigma_{i}\right)$ a normalized version of $x_{i} ; \bar{x}_{i}$ and $\sigma_{i}$ are the mean and standard deviation of attribute $i$. This update procedure has time complexity $O(m)$, which makes it very suitable for dealing with high-speed data streams; furthermore, the Delta rule is known to converge even if the data points do not represent a straight line.

\section{The iRetis algorithm}

The learning algorithm we propose is similar to FIMT, except for two important points. First, while FIMT finds the split that maximally reduces standard deviation, like M5 [7], our method tries to minimize the residual variance after learning a linear model in both subtrees, which makes more sense into model tree learning setting (but is also computationally more complex). Second, while FIMT gradually approaches the optimal regression line using perception learning, our approach at each point in time computes the least-squares regression function for the data seen until then. Again, this is computationally more complex, but may enable the learner to reach comparable levels of accuracy with fewer required observations. We next discuss these differences in more detail. 


\subsection{The Mauve and Retis heuristics}

The quality measure of FIMT's splitting criterion is based on standard deviation reduction. Even if the observed data points are perfectly linear, the SDR will be non-zero, which indicates that splitting a leaf node can improve the overall model. Vens et. al proposed a splitting criterion based on regression called Mauve [10]. The Mauve heuristic inspects the standard deviation of the residuals after performing linear regression, rather than the attribute values. A simple linear regression (i.e., using one predictor variable) is performed, using as predictor the attribute for which a split is being considered. A model is built for the leaf node as well as the two hypothetical children that arise from the split. As a result, the splits chosen by Mauve are better adapted to the fact that the leaves will contain linear models, not constants.

By expanding the statistics that are kept in the E-BST structure with the sum of attribute values $x_{j}$, the sum of squared attribute values $x_{j}^{2}$ and the sum of the product of attribute and target values $x_{j} y$, it is possible to calculate the residual standard deviation for simple regression on attribute $j$ at any time. Let $e_{i}$ be the residual for example $i$ and $\bar{e}$ be the residual mean. The residual standard deviation in a leaf model $l$ is:

$$
R S D(l)=\sqrt{\frac{1}{N} \sum_{i=1}^{N}\left(e_{i}-\bar{e}\right)^{2}} .
$$

Since Mauve considers simple regression, the model expression for an attribute $x_{j}$ will be of the form $\hat{y}=a+b x$. Using the substitution $e_{i}=\hat{y}_{i}-y_{i}$ in Equation (6), the residual standard deviation becomes:

$$
R S D(l)=\sum_{i} y_{i}^{2}-\frac{1}{N}\left(\sum_{i} y_{i}\right)^{2}-\frac{\left(\sum_{i} x_{i} y_{i}-\frac{1}{N} \sum_{i} x_{i} \sum_{i} y_{i}\right)^{2}}{\sum_{i} x_{i}^{2}-\frac{1}{N}\left(\sum_{i} x_{i}\right)^{2}}
$$

Since this method considers only the split attribute in its regression, it is possible that some components of the target function will not be modeled. We can extend the Mauve heuristic to multiple linear regression. In that case we are effectively using the RETIS heuristic [6]. We will refer to this algorithm as iRetis. In this case, the residual for an example $i$ can be written as:

$$
e_{i}=\beta_{0}+\beta \mathbf{x}_{i}-y_{i}
$$

Substituting the new definition of the residual in Equation (6), we get the following expression for the residual standard deviation $R S D$ and residual variance $R V a r$, using complete linear regression in $m$ attributes: 


$$
\begin{aligned}
R \operatorname{Var}(l)= & \frac{1}{N}\left(\sum_{i}\left(\beta \mathbf{x}_{i}\right)^{2}+\sum_{i} y_{i}^{2}-2 \sum_{i}\left(\beta \mathbf{x}_{i} y_{i}\right)\right) \\
& -\frac{1}{N^{2}}\left(\left(\sum_{i} \beta \mathbf{x}_{i}\right)^{2}+\left(\sum_{i} y_{i}\right)^{2}-2 \sum_{i} y_{i} \sum_{i} \beta \mathbf{x}_{i}\right) \\
R S D(l)= & \sqrt{R \operatorname{Var}(l)}
\end{aligned}
$$

From this equation we can deduce the statistics we need to keep in order to calculate the residual standard deviation incrementally. The most obvious statistics are the number of observed data points $N$, the sum of $y$ values and the sum of squared $y$ values. The term $\beta \mathbf{x}_{i}$ can be expanded to $\sum_{j=1}^{m} \beta_{j} x_{i, j}$. By re-arranging the sums we observe

$$
\sum_{i} \beta \mathbf{x}_{i}=\sum_{j} \beta_{j} \sum_{i} x_{i, j}
$$

revealing the need to keep track of the sums of attribute values. Finally we observe the term $\sum_{i}\left(\beta \mathbf{x}_{i}\right)^{2}$. By expanding the term $\beta \mathbf{x}_{i}$ as above we get:

$$
\sum_{i}\left(\beta \mathbf{x}_{i}\right)^{2}=\sum_{i}\left(\sum_{j}\left(\beta_{j} x_{i, j}\right)^{2}+2 \sum_{j<k} \beta_{j} x_{i, j} \beta_{k} x_{i, k}\right) .
$$

This last equation shows the need to keep track of the sum of squares of attribute values, and the sums of the products of all combinations of attribute values.

The amount of statistics kept for each split is $\frac{m^{2}}{2}+3 m+3$. Like FIMT, these statistics are kept in an E-BST structure. There is one such structure for each attribute. Every E-BST grows by one node with each data point. This brings the total amount of statistics kept during algorithm execution to $m n\left(\frac{m^{2}}{2}+3 m+3\right)$. This is a higher count than just storing each data point, however this structure allows us to immediately calculate the residual standard deviation reduction for every split, for a complete linear regression. Additionally it allows us to instantiate the weights of the linear models in the child nodes of a split to the least squares estimate immediately. This contrasts with FIMT, where the weights of child nodes are set equal to the weights of the parent node after a split, thus needing additional data points to converge to their new values. After a split is made, the E-BST structure for that attribute is discarded, freeing up the memory it occupied.

\subsection{Incremental linear regression}

While FIMT's perceptron update rule has a low time complexity for a single update, a large number of updates is required for the perceptron to converge. After each split, the amount of data points arriving in the children of that split 
is halved on average. As the tree grows larger, the individual perceptrons in the leaves observe ever fewer data points, causing them to converge very slowly in later stages of learning. Therefore we propose a method based on linear least squares regression to estimate the weights of the linear models in the leaves. The least squares estimation of the weights is $\hat{\beta}=\left(\mathbf{x}^{T} \mathbf{x}\right)^{-1} \mathbf{x}^{T} y$.

The elements of $\mathbf{x}^{T} \mathbf{x}$ and $\mathbf{x}^{T} y$ can be updated incrementally, as each data point is observed. Therefore it is possible to have a least squares estimate of the weight vector at any time during learning. It is important to note that the elements of both matrices all appear in the necessary statistics for calculating the residual standard deviation described in the previous section. As a result, there is no need to explicitly keep these matrices in memory.

To acquire this estimate, it is necessary to invert the matrix $\mathbf{x}^{T} \mathbf{x}$ and multiply it with $\mathbf{x}^{T} y$. The time complexity of the matrix inversion and matrix multiplication steps is $O\left(\mathrm{~m}^{3}\right)$. Slightly faster multiplication and inversion algorithms exist, however they are not used here since they only provide an advantage on very large matrices. This time complexity is significantly slower than FIMT's $O(m)$ perceptron update rule. However, for relatively small values of $m$, this difference might not be troublesome.

\section{Experiments}

We experimentally compare iRetis, iMauve and FIMT in terms of the size of the induced model tree, the execution speed and accuracy. We investigate the following hypotheses:

i iRetis and iMauve learn smaller trees compared to FIMT,

ii learning smaller trees will not result in loss of accuracy, thanks to the improved splitting heuristic,

iii iRetis and iMauve require fewer data points to reach the same level of accuracy as FIMT, because they choose splits more intelligently and immediately instatiate linear models in the child nodes of a split with the proper least squares estimate

\subsection{Setup}

We took eleven datasets from Luís Torgo's collection of datasets [9] and the UCI machine learning repository [1]. Data for the CART dataset is drawn from two distinct hyperplanes, with noise added. We also added a noiseless version of this dataset; iRetis should build a model with zero error on such a dataset.

On each dataset a 10-fold cross-validation was performed. The same folds were used for each algorithm. During training, each algorithm received data samples from the training set in the same order. The results listed in Tables 1 and 2 are the average values over the 10 folds. The metrics listed are relative to FIMT's performance (i.e. FIMT's score is 1 for all metrics for all datasets). The plots shown in Figures 1 through 4 were generated by taking the average RRSE at each evaluation point over the 10 folds. 


\subsection{Results}

Table 1 shows the end results for all datasets, as well as the number of attributes and examples in those datasets. The average rank for each algorithm over the datasets is shown at the bottom of the tables. To test the performance of iRetis, we use the Friedman test followed by the Bonferroni-Dunn post-hoc analysis. The null hypotheses in the Friedman test is that all algorithms have equal performance. If the Friedman test statistic is larger than a critical value, determined by the amount of algorithms and the amount of datasets, the null hypotheses can be rejected and we can follow up with the post-hoc analysis. The BonferroniDunn post-hoc analysis defines a critical distance for the average ranks of two algorithms. If the difference in average ranks is more than this critical distance, the difference can be regarded as significant.

The critical value for the Friedman test with 3 treatments (the algorithms) on 12 blocks (the datasets) is $Q_{0.05}^{*}=6.500$ for a confidence level $\alpha=5 \%$. The critical distance at this confidence level is $C D=0.879$.

For the tree size metric, we calculate $Q=10.17>Q_{0.05}^{*}$, therefore we reject the null hypothesis. Since the differences in average rank of iRetis compared to the other algorithms is greater than $C D$, we can conclude that iRetis tends to find smaller trees than the others. The difference between iMauve and FIMT is not significant.

In terms of runtime, we observe no differences in rank between the datasets: FIMT is always the fastest, and iRetis is always the slowest. This result is significant at $\alpha=0.05$.

We measured accuracy by means of RRSE. The $Q$-statistic for the RRSE results is $Q=8.17>Q_{0.05}^{*}$. We can reject the null hypothesis. The differences in average rank of iRetis compared to the other algorithms is greater than $C D$, therefore we can conclude that iRetis converges on more accurate trees than the other systems. The difference between iMauve and FIMT is again not significant.

These results confirm hypotheses i and ii. Figures 1 through 4 show how test set accuracy (measured by RRSE) evolves with the number of training samples seen by the algorithms. Each point on this plot is the average RRSE over the 10 runs of the algorithm, measured after the corresponding amount of data points observed. Due to space constraints, we cannot show the plots for all datasets tested, but on most datasets, iRetis clearly learns more accurate models from fewer datapoints, as in Figures 1 and 2. There are some datasets however, where all methods seem to struggle (shown in Figures 3 and 4), and the difference is less clear.

To further investigate the impact of the longer computation time of iRetis, we have simulated a data stream providing 200 samples per second. This sampling rate was determined by investigating the rate at which iRetis processes samples, and choosing a rate high enough to cause iRetis to drop samples. Here, arriving examples are discarded if the previous example has not yet been fully processed. Table 2 shows the results for this experimental setup. As with the previous experiment, the values for RRSE, tree size an runtime are metrics relative to FIMT. Additionally we included the dropped sample rate (DSR), this is the 
Table 1. Results for RRSE, Tree Size and runtime . Additionally the number of attributes $d$ and the number of samples $N$ is shown for each dataset.

\begin{tabular}{lcccccccc}
\hline & & & \multicolumn{3}{c}{ iRetis } & \multicolumn{3}{c}{ iMauve } \\
& $d$ & $N$ & RRSE & Size & Time & RRSE & Size & Time \\
\hline Friedmann & 10 & 40768 & $\mathbf{0 . 5 6 6}$ & $\mathbf{0 . 7 1 6}$ & 7.619 & 0.735 & 0.862 & 1.198 \\
Lexp & 5 & 4000 & $\mathbf{0 . 4 7 1}$ & $\mathbf{0 . 6 8 8}$ & 8.012 & 0.875 & 0.907 & 1.251 \\
Cal housing & 8 & 20640 & 1.100 & $\mathbf{0 . 7 6 5}$ & 4.508 & 1.144 & 1.084 & 1.106 \\
CART & 10 & 40768 & 1.033 & 0.794 & 1.707 & 2.401 & $\mathbf{0 . 1 4 6}$ & 1.622 \\
Losc & 5 & 4000 & $\mathbf{0 . 6 3 7}$ & $\mathbf{0 . 8 4 7}$ & 4.824 & 0.697 & 0.904 & 1.239 \\
Paraboloid & 12 & 4000 & $\mathbf{0 . 1 6 8}$ & $\mathbf{0 . 9 0 7}$ & 2.166 & 0.215 & 1.035 & 1.269 \\
CARTNoiseless & 10 & 4000 & $\mathbf{0 . 0 0 0}$ & 0.365 & 1.939 & 11.883 & $\mathbf{0 . 2 3 2}$ & 1.565 \\
Ailerons & 40 & 13750 & $\mathbf{0 . 1 8 3}$ & $\mathbf{0 . 7 2 7}$ & 5.087 & 0.197 & 1.580 & 1.032 \\
PoleTelecom & 26 & 15000 & $\mathbf{0 . 2 7 9}$ & $\mathbf{0 . 5 8 6}$ & 4.811 & 0.501 & 1.036 & 1.200 \\
CPU & 21 & 8192 & $\mathbf{0 . 6 4 2}$ & $\mathbf{0 . 8 6 4}$ & 15.173 & 2.317 & 1.515 & 1.115 \\
Physicochemical & 9 & 45730 & 1.094 & $\mathbf{0 . 7 3 4}$ & 5.105 & 1.052 & 1.009 & 1.107 \\
Abalone & 8 & 4177 & $\mathbf{0 . 9 2 8}$ & 1.059 & 4.662 & 1.094 & 1.507 & 1.144 \\
\hline Avg Rank & & & $\mathbf{1 . 3 3}$ & $\mathbf{1 . 2 5}$ & 3 & 2.42 & 2.42 & 2 \\
Avg Rank FIMT & & & $(2.25)$ & $(2.33)$ & $(\mathbf{1})$ & $(2.25)$ & $(2.33)$ & $(\mathbf{1})$ \\
\hline
\end{tabular}

proportion of samples that was dropped relative to the total amount of samples in the dataset. The $Q$-values are still larger than $Q_{0.05}^{*}$ for RRSE, tree size and runtime. Additionally, we observe that the difference in ranks between iRetis and the other approaches is still larger than the critical distance. Keep in mind that the results in Tables 1 and 2 can not be compared directly. The performance of FIMT will be different, and hence the normalization factor is different as well. We conclude that even if samples are dropped during learning, iRetis still finds more accurate trees.

\section{Conclusions}

We have investigated to what extent incremental model tree learners may benefit from more complex update procedures. To this aim we implemented two variants of the current state-of-the-art system FIMT, called iRetis and iMauve. These use the same EBST structure to manage statistics about the data, and the same Hoeffding bound to decide the best split points, as FIMT, but they use a heuristic based on linear regression (simple for iMauve, multiple for iRetis), and use closed formulas for least-squares regression instead of a perceptron.

Given the more complex splitting heuristic, we expected iMauve to perform better than FIMT. Surprisingly, the average rank for iMauve was slightly worse than FIMT's. However this difference was too small to be of statistical significance, and we can not draw any conclusions. 
Table 2. Results for the data stream simulation experiment.

\begin{tabular}{lcccccccc}
\hline & \multicolumn{4}{c}{ iRetis } & \multicolumn{4}{c}{ iMauve } \\
& RRSE & Size & Time & DSR & RRSE & Size & Time & DSR \\
\hline Friedmann & $\mathbf{0 . 5 6 7}$ & $\mathbf{0 . 5 9 9}$ & 8.379 & 0.177 & 0.734 & 0.860 & 1.204 & 0.000 \\
Lexp & $\mathbf{0 . 5 4 3}$ & $\mathbf{0 . 5 5 3}$ & 13.857 & 0.091 & 0.875 & 0.907 & 1.249 & 0.000 \\
Cal housing & 1.072 & $\mathbf{0 . 7 1 9}$ & 6.337 & 0.064 & 1.143 & 1.083 & 1.073 & 0.000 \\
CART & 1.033 & 0.794 & 2.826 & 0.000 & 2.401 & $\mathbf{0 . 1 4 6}$ & 2.582 & 0.000 \\
Losc & $\mathbf{0 . 6 3 7}$ & $\mathbf{0 . 7 8 6}$ & 7.516 & 0.043 & 0.697 & 0.904 & 1.279 & 0.000 \\
Paraboloid & $\mathbf{0 . 1 7 1}$ & $\mathbf{0 . 9 0 7}$ & 3.296 & 0.002 & 0.215 & 1.035 & 1.132 & 0.000 \\
CARTNoiseless & $\mathbf{0 . 0 0 0}$ & $\mathbf{0 . 3 6 5}$ & 4.155 & 0.000 & 11.883 & 0.232 & 2.709 & 0.000 \\
Ailerons & $\mathbf{0 . 1 7 9}$ & $\mathbf{0 . 3 5 6}$ & 3.662 & 0.501 & 0.198 & 1.586 & 1.208 & 0.000 \\
PoleTelecom & 0.740 & $\mathbf{0 . 5 0 8}$ & 6.833 & 0.190 & $\mathbf{0 . 7 1 5}$ & 1.047 & 1.725 & 0.000 \\
CPU & $\mathbf{0 . 7 4 9}$ & $\mathbf{0 . 4 5 6}$ & 7.906 & 0.491 & 2.074 & 1.444 & 1.137 & 0.001 \\
Physicochemical & 1.158 & $\mathbf{0 . 6 5 1}$ & 6.135 & 0.112 & 1.053 & 1.006 & 1.095 & 0.000 \\
Abalone & $\mathbf{0 . 9 4 6}$ & 1.029 & 6.587 & 0.056 & 1.094 & 1.507 & 1.104 & 0.000 \\
\hline Avg Rank & $\mathbf{1 . 4 1 7}$ & $\mathbf{1 . 2 5}$ & 3 & & 2.33 & 2.42 & 2 & \\
Avg Rank FIMT & $(2.25)$ & $\mathbf{( 2 . 3 3 )}$ & $\mathbf{( 1 )}$ & & $(2.25)$ & $(2.33)$ & $(\mathbf{1})$ & \\
\hline
\end{tabular}

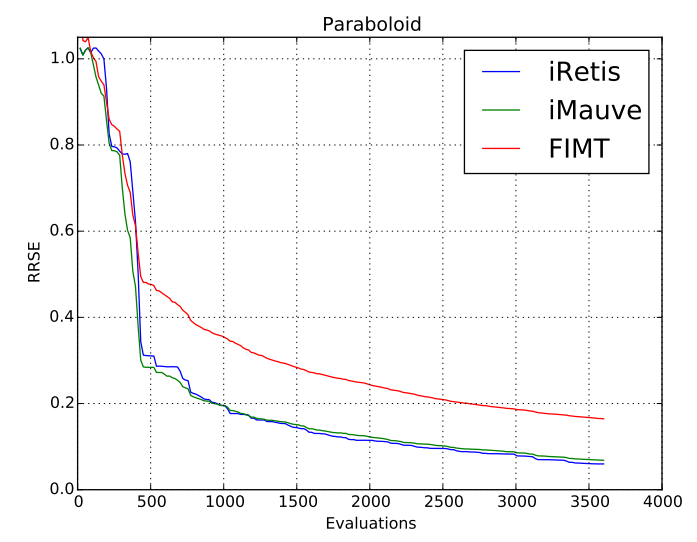

Fig. 1. Results for Paraboloid 


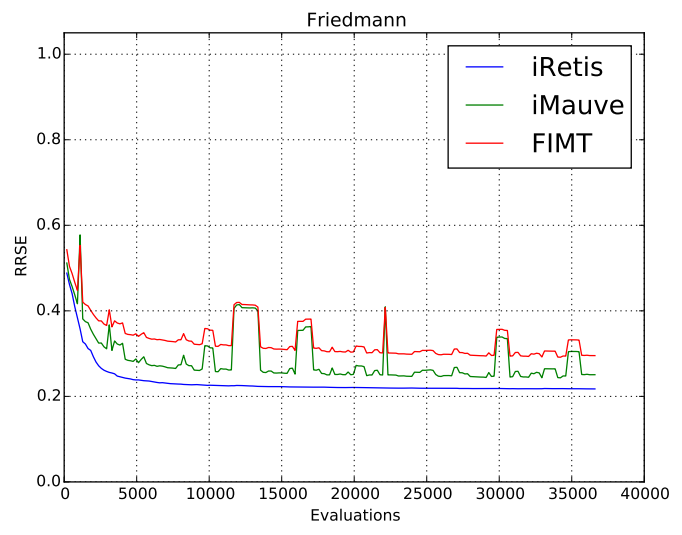

Fig. 2. Results for Friedmann

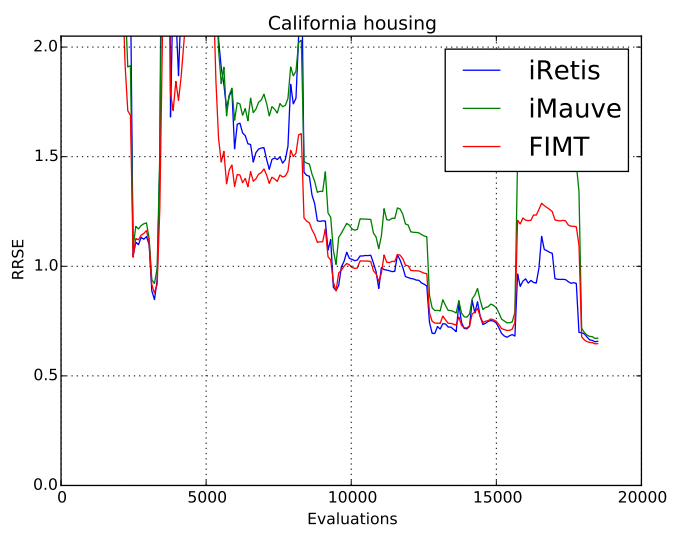

Fig. 3. Results for Cal housing

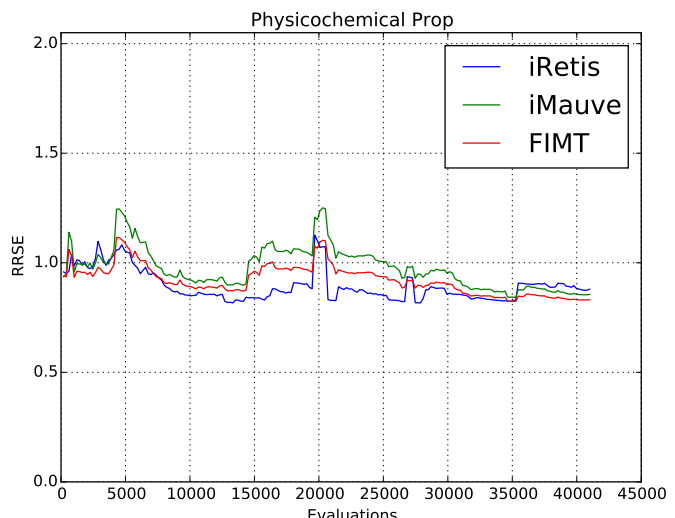

Fig. 4. Results for Physicochemical 
We found that on datasets with a relatively small number of attributes iRetis builds more compact trees, with comparable or even superior accuracy, compared to iMauve and FIMT. In terms of the number of examples seen, these models are also obtained sooner, even when some examples have to be skipped because they arrive too fast. In other words, slower in terms of computation can still be faster in terms of actual time, under certain realistic conditions such as relatively low dimensionality and allowed computation time between examples in the order of milliseconds or higher.

These results show that updating the model more carefully can pay off, even if it comes at the expense of number of data points that can be observed. More detailed research is needed to identify the conditions under which a more complex update procedure is warranted.

\section{Acknowledgements}

DV was supported by the Research Foundation Flanders (FWO-Vlaanderen), projects G.0255.08 and G.0179.10.

\section{References}

1. Bache, K., Lichman, M.: UCI machine learning repository (2013), http:// archive.ics.uci.edu/ml

2. Breiman, L., Friedman, J., Olshen, R., Stone, C.: Classification and Regression Trees. Wadsworth and Brooks, Monterey, CA (1984)

3. Domingos, P., Hulten, G.: Mining high-speed data streams. In: Proceedings of the Sixth ACM SIGKDD International Conference on Knowledge Discovery and Data Mining. pp. 71-80. KDD '00, ACM, New York, NY, USA (2000)

4. Hoeffding, W.: Probability inequalities for sums of bounded random variables. Journal of the American Statistical Association 58(301), 13-30 (March 1963), http://www . jstor.org/stable/2282952?

5. Ikonomovska, E., Gama, J., Džeroski, S.: Learning model trees from evolving data streams. Data Mining and Knowledge Discovery 23(1), 128-168 (2011)

6. Karalič, A.: Employing linear regression in regression tree leaves. In: Proceedings of the 10th European Conference on Artificial Intelligence. pp. 440-441. ECAI '92, John Wiley \& Sons, Inc., New York, NY, USA (1992), http://dl.acm.org/ citation. cfm?id=145448.146775

7. Quinlan, J.R.: Learning with continuous classes. In: Proceedings of the Australian Joint Conference on Artificial Intelligence. pp. 343-348. World Scientific, Singapore (1992)

8. Quinlan, J.R.: C4.5: Programs for Machine Learning. Morgan Kaufmann Publishers Inc., San Francisco, CA, USA (1993)

9. Torgo, L.: Regression datasets (Sep 2014), http://www.dcc.fc.up.pt/ Itorgo/ Regression/DataSets.html

10. Vens, C., Blockeel, H.: A simple regression based heuristic for learning model trees. Intell. Data Anal. 10(3), 215-236 (May 2006) 